\title{
Gait Recognition System Using Gabor Wavelet and Active Gait Differential Image
}

\author{
${ }^{1}$ Ojo, John Adedapo, ${ }^{2}$ Afolabi, Simeon Olukayode \\ Department of Electronic and Electrical Engineering, \\ LadokeAkintola University of Technology, \\ P. M. B. 4000, Ogbomoso, Oyo State, Nigeria. \\ ${ }^{1}$ jaojo@lautech.edu.ng; ${ }^{2}$ simeonolukayode@gmail.com
}

\begin{abstract}
Gait is a behavioural biometric process that serves to identify persons using their walking style. It is un-obstructive, not easy to conceal and offers distance recognition. Various approaches have been employed to improve the performance and accuracy of gait biometric systems but the performance is yet to measure up to that of other biometric recognition systems. In this work, Gabor wavelets were used to extract Active Gait Differential Image (AGDI) features, while Principal Component Analysis (PCA) was used for feature dimensionality reduction. The classification was performed using Support Vector Machine (SVM) and silhouette images from Chinese Academy of Science Institute of Automation (CASIA) gait dataset was used for testing. The performance was evaluated using accuracy, equal error rate, false acceptance rate and false rejection rate and it gave $99.19 \%, 1 \%, 0 \%$, and $2 \%$ respectively for the metrics used.
\end{abstract}

Keywords: Gait, Active Gait Differential Image (AGDI), Support Vector Machine (SVM), Principal Component Analysis.

\section{Introduction}

Motivated by the need for automated person identification systems in areas that are security-sensitive such as airports, banks, shopping malls and parking lots, identification of humans at a distance has received growing interest in recent times. Video-based human motion analysis is able detect, track and identify people and understudy behaviour of humans from image sequences. Gait is an example of such behavioural biometric that can be noticed and used to identify people from a distance. It is recognizing people through their style of walking, an approach relatively new compared to other biometrics such as fingerprints, iris, palm, and facial [1].

Gait recognition system can be classified to three groups based on the type of sensors used, which are: Floor Sensor Based System [2], Wearable Sensor-based Approach [3] and Video Sensor-based Gait Approach [2]. This work explores the video sensor-based gait analysis approach for our gait recognition system. The framework of a Video sensor-based gait recognition system is shown in Figure 1. 


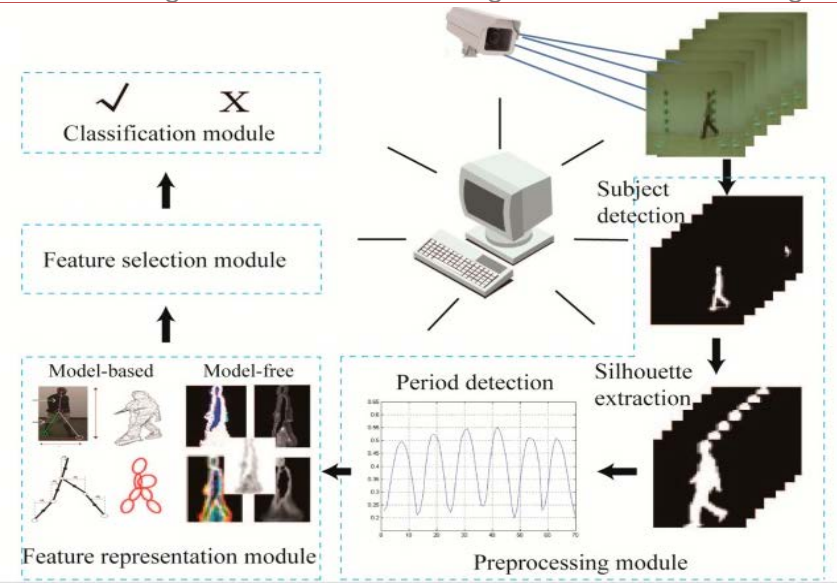

Figure 1: Video-based gait recognition framework [2].

Gait analysis could be model-based or model-free method. In model-based approaches, the structure or motion of the human body is modelled, then features that are matched to the modelled components are extracted. The features contain a combination of information from the shape and dynamics of human gait. Model-free methods also known as silhouette analysis the gait motion directly to identify individuals and does not require a fore knowledge of the gait model. Statistical features from the entire silhouette image without assumption of the underlying structure are extracted. A common method of extracting gait features from silhouette of gait subjects is by measuring body parameters of the subject on a frame by frame basis. Others include, feature image templates, Radon Transform and Canonical Transform. However, some of these feature images fail to preserve the static and dynamic components of walking humans and thus reduces the accuracy of the recognition systems. Therefore, there is the need to construct gait feature image that preserves both static and dynamic components of gait to build human-like recognition system. This work explores the uniqueness of the walking pattern of an individual as a biometric tool using features extracted by Gabor wavelets on Active Gait Differential Image.

The framework of the video sensor based gait recognition system comprises four modules, viz: Preprocessing module which involves detection of subject and extraction of silhouette from the original video, feature representation module, feature selection module and classification module.

\section{Literature Review}

Previous works in gait recognition adopted several ways of feature extraction, feature representation, feature dimentionality reduction and classification. In building an efficient gait recognition system, some of the key factors to consider include: type of features extracted, method by which the features are extracted, feature dimentionality reduction method, means of classification and consideration of static and dynamic components of gait of the individual subjects. Earlier work in the field of gait employed the use of various devices to determine the gait of a person. The early work of [4] on human motion perception corroborates this point. [4] adopted the use of Moving Light Display (MLD) device. In the experiment, movement of bright spot on the display created and impression of walking, and running to the viewers. This author finally demonstrated that a dynamic array of point light is sufficient to recognize the presence of a 'walker' and went further that the same array is sufficient for the recognition of a particular 'walker'. The work of [5] is an upshot of Johannson work. This authors' experiment is a refinement of the early work of [5] as [5] explored the use of optical device for gait recognition. Gait subjects were tracked by region growing followed by background subtraction. From each silhouette image, spatial (centroid of each silhouette window) and temporal template with U- 
flow (horizontal component of optical flow field), V-flow (vertical component of flow) and U, V-flow (magnitude of $\mathrm{U}, \mathrm{V}$ ) were extracted. $100 \%$ recognition rate of test sequence was achieved in Canonical space after projection. It was however noted by this author that optical flow technique is sensitive to changes in video frame rate and speed of subjects. [6] employed the use of a device called Continuous Wave Rader (CWR) for human gait recognition. Signals reflected from torso, leg and arm of gait subjects were used as gait features when CRW was incident on a person walking towards or away from the radar. A simple binary classifier was developed that classified detection as either a person is present or not present. The classifier result gave a correct classification rate of $80 \%$, leakage rate of $12 \%$ and false alarm rate of zero (0\%). However, the shortcoming of this method is that it cannot distinguish (discern) whether the subject is moving towards or away from the radar. Further to the method that employed the use of devices to determine gait is another approach known as silhouette based gait recognition approach. This method serves to extract gait features from binary silhouette image of gait subject. Features are extracted by measuring different body parameters of gait subject. [7] extracted four static body parameters directly from the silhouette of gait subjects namely, the body height, torso length, leg length and step length and used them for person identification. It was noted that the method for estimating step length does not explore the periodicity of walking, as a result making it sensitive to tracking and camera calibration errors. In a somewhat similar manner, [8] after tracking the gait subject using background subtraction method with a 2D position of a silhouette, employed stride length and cadence as measured from individual gait subjects as gait features while the Bayesian rule was employed as classifier achieving a recognition rate of $40 \%$. It was observed that the non-inclusion of static body parameters (static components of gait) in the algorithm made the recognition rate to drop compared with [7]. This is an indication that static components of gait enhance the accuracy of gait recognition system. [9] estimated the periodicity in gait sequences obtained from all three (3) views (lateral, oblique and frontal) with respect to the image plane. Gait cycle, differences between bounding box and silhouette (distance vector) were taken as gait features, while eigenspace transformation based on Principal Components Analysis was used for dimensionality reduction. The algorithm gave an encouraging result but covariate factor like changes in clothing was not catered for in the experiment. The algorithm developed by [10] gave $96.3 \%$ recognition rate when gait cycle, silhouette height, and width, and gait volume were features extracted from different gait subjects through CCTV cameras that present subject from upper front view, while K-nearest neighbour classifier was employed as the classifier. It was however noted that the system performed better in lateral view of gait subjects.

Gait recognition work has witnessed tremendous improvement over the years which have brought about a drift from the silhouette based method to Feature Image based approach. Recent work in gait serves to mathematically combine silhouette images of gait subjects into a single image template called feature image template. This eliminates the 'rigor' of having to extract features on a frame by frame basis from silhouette image of a subject whose gait is to be determined. Examples of feature image include, Gait Energy Image, Frame Differential energy Image, Gait Energy Volume, Active Gait Energy Image and so on. [11] considered feature selection on Gait Energy Image. Supervised and unsupervised feature selection methods were used to extract static and dynamic information of gait subjects while Principal Component Analysis and Median Discriminant Analysis were applied to the selected features for dimensionality reduction. Conclusion was drawn that unsupervised learning feature is advantageous and affords higher recognition rate and lesser computational cost over supervised one. [12] used a new gait representation called Frame Difference Energy Image (FDEI) as against the Gait Energy Image (GEI) employed by [11]. Frieze and wavelet features were extracted from original silhouette image and FDEI representation respectively, while Hidden Markov Model 
Ojo, J ohn Adedapo, Afolabi, Simeon Olukayode; Gait Recognition System Using Gabor Wavelet and Active Gait Differential Image. Advances in Image and Video Processing, Volume 5 No 4, August (2017); pp: 1-10

HMM was used for recognition. This approach could take care of the problem of silhouette incompleteness and occlusion which imparts negatively on recognition. Also, both static and dynamic information of gait subjects were captured in the FDEI representation. The results observed were better than the methods using binary image and Gait Energy Image for recognition.

[13] worked on gait recognition where GEI was constructed and PCA with and without Radon Transform (RT) were applied on the feature image (GEI). RT and PCA were said to have performed the same roles feature extraction and feature dimensionality reduction respectively. An improved recognition system was observed using Carnegie Melon University (CMU) MOBO dataset for gait experiment. The two techniques achieved EER of $94.23 \%, 82,28 \%$ and $90.38 \%$ using PCA only and $96.15 \%, 82.7 \%$ and $92.3 \%$ for PCA with RT for a slow walk, fast walk, and carrying ball walking conditions respectively.

Feature image template such as Gait Energy Images (GEIs) and its modifications form the basis of many recent appearance based gait recognition systems. [14] proposed an extension of GEI used by [13] to operate in the three-dimensional domain, using binary voxel volumes instead of 2D silhouettes. This proposed Gait Energy Volume (GEV) algorithm showed an improvement over 2D silhouette image in that GEV circumvents the issue of view dependency, as well as having no pose ambiguity, no selfocclusion and allowing easier segmentation of unwanted regions. GEV is computed using

$$
G E V(K)=\frac{1}{n} \sum_{t=1}^{n} V(t)
$$

where $\mathrm{n}$ is the number of frames in the $\mathrm{K}^{\text {th }}$ and $\mathrm{V}$ is the aligned voxel volumes.

Features were extracted from the lower part of human MDA was used to extract the effective discriminant features while PCA was employed for dimensionality reduction. Euclidean distance was used for similarity measure between the probe and gallery sequence. This method was evaluated on CMU Mobo database and the in-house captured frontal depth-based gait database. It was however revealed that GEV required a more complex hardware setup, making it impractical for many applications. It is expected that an efficient feature image template with effectual feature extraction and classification procedure would ensure a more accurate gait recognition system. With this background, to build an efficient gait recognition system, there is need to construct a feature image template that contains rich gait information and employ a feature extraction technique that is derived in harmony with the physical world which is suitable for building human-like recognition systems owing to its biological significance. This work explored the biological significance of Gabor wavelets and the strength of Active Gait Differential Energy Image for building a more efficient gait recognition system.

\section{Methodology}

\subsection{Overview of the Developed System}

Figure 2 shows the block diagram of the developed gait recognition system, which comprises the preprocessing, feature extraction, feature dimensionality reduction and the classification stages.

The developed gait recognition system was tested with Chinese Academy of Science Institute of Automation (CASIA) gait database. The dataset contains videos of 124 subjects (93 males and 31 females) captured from 11 view angles (ranging from $0^{0}$ to $180^{\circ}$ with view angle interval of $18^{0}$ ). It is made up of six normal walking sequences for each subject (normal 01, ..., normal 06) conducted from every view angle and there are 3-8 gait cycles of about 40-100 frames for every walking sequence. The 
video frame size is $320 \times 240$ pixels, and the frame rate is $25 \mathrm{fps}$. Figure 3 shows twenty-six (26) images of raw silhouette of one subject from the CASIA gait database, showing various positions of gait while walking. These twenty-six images constitute two gait cycles, which is further divided into two phases namely the stance phase (when the foot is in contact with the ground) and the swing phase (the period when the foot is in the air). From the raw silhouette in Figure 3, images labelled one (1) to thirteen (13) constitute one gait cycle while images labelled fourteen (14) to twenty-six (26) depicts another cycle of gait. It was observed that for each gait cycle, the position of the silhouette (walking individual) at the beginning of the walking process (which denotes the beginning of a cycle) is the same as what is obtainable at the end of the cycle. Active Gait Differential Image was constructed by accumulating the differences between every two frames of the silhouette images as shown in Figure 4. This image contains the Spatial (static) and Temporal (dynamic) components of human gait. The lower and higher intensity regions in the image represent the static and dynamic components of gait respectively.

In the pre-processing stage, effect of distance was removed from the gait images. Every silhouette image was normalized to equal height by employing bicubic interpolation. This helped in ensuring that we have a smoother silhouette image.

The centroid $(x, y)$ of a silhouette is defined as follows

$$
x_{c}=\frac{1}{n} \sum_{i=1}^{n} x_{i}, \quad y_{c}=\frac{1}{n} \sum_{i=1}^{n} y_{i},
$$

where $\mathrm{n}$ is the number of pixels in the silhouette.

Suppose that $I_{j}$ and $I_{j+1}$ are two adjacent images aligned by the centroid, the gait differential image $D_{j}$ can be defined as

$$
D_{j}(x, y)=\begin{aligned}
& 0 \text { if } \mathrm{I}_{j}(x, y)=I_{j+1}(x, y) \\
& 1 \text { if } \mathrm{I}_{j}(x, y) \neq I_{j+1}(x, y)^{\prime}
\end{aligned},
$$

where $\mathrm{j}$ is the frame number in the image sequence and $X$ and $y$ are the values in the 2D image coordinate. 


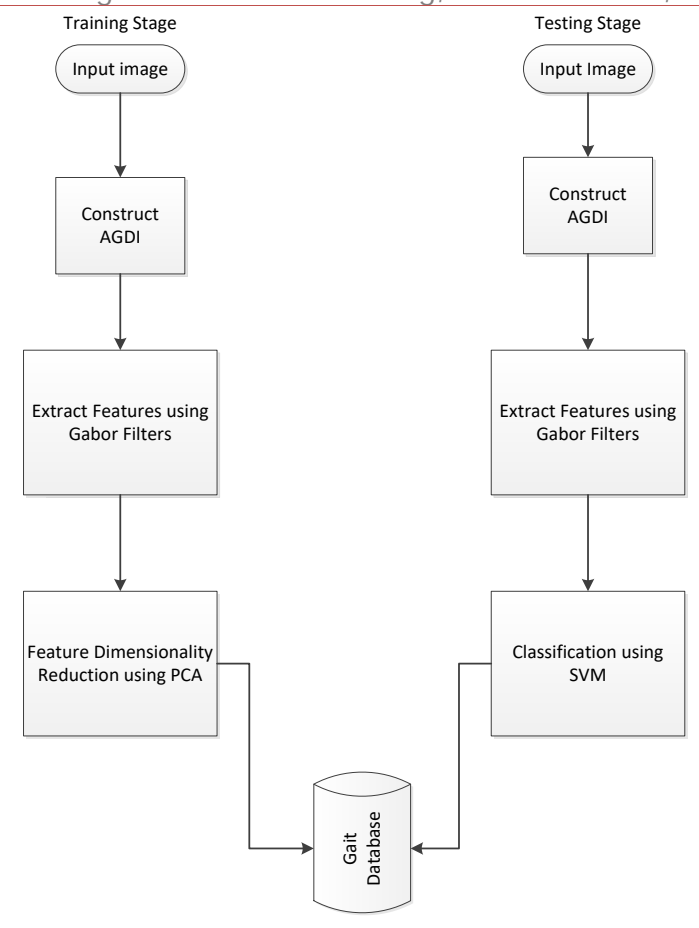

Figure 2: Block diagram of the Developed Gait Recognition System

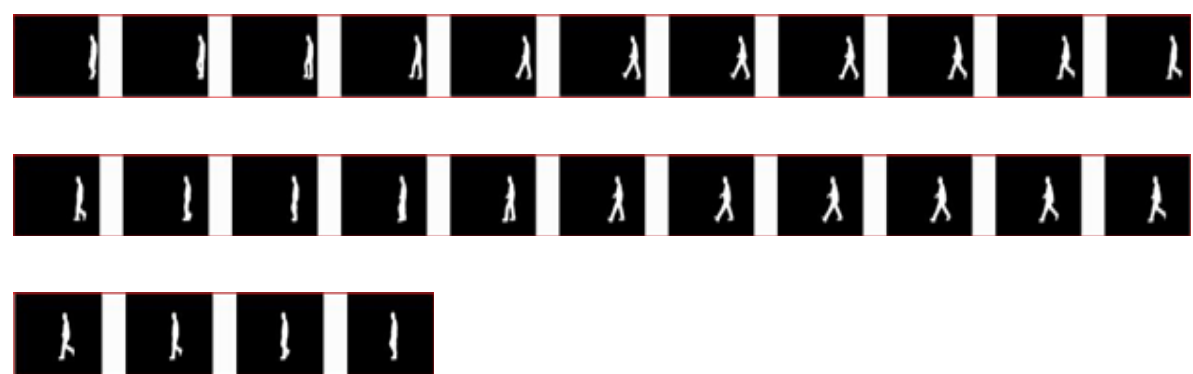

Figure 3. Raw Silhouette Images

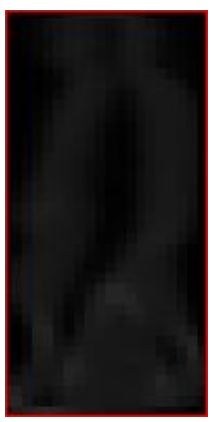

Figure 4 Active Gait Differential Image

By overlapping all the differential image of one human gait cycle, the average gait differential image was obtained from:

$$
G(x, y)=\frac{1}{N-1} \sum_{j=1}^{N-1} D_{j}(x, y),
$$

where $\mathrm{N}$ is the number of frames in the complete gait cycle(s) of a silhouette sequence [16].

In the training and testing stages, Gabor wavelets was performed on the constructed AGDI to extract discriminative features, Principal Component Analysis (PCA) was used for feature dimensionality 
reduction, while Support Vector Machine (SVM) was employed for classification of the stored features.

Gabor function acts as a low-level oriented edge and texture discriminators, and is sensitive to different frequencies and scale information. Mathematically, a 2D Gabor function, $g$, is the product of a 2D Gaussian and a complex exponential function. The general expression is given by

$$
g_{\theta, \sigma, \lambda}(x, y)=\exp \left[-\frac{1}{2}(x, y) M(x, y)^{T}\right] \exp \left[\frac{j \pi}{\lambda} \cos \theta+\mathrm{ysin} \theta\right]
$$

where $\theta$ represents the orientation, $\lambda$ is the wavelength, and $\sigma$ represents scale at orthogonal directions.

By selectively changing each of the Gabor function parameters the filter can be tuned to a pattern arising in the images. The Gabor filter responds by convolving a Gabor function with image patterns I $(x, y)$, which helps to evaluate their similarities. Gabor response at point $\left(x_{o}, y_{o}\right)$ is defined as

$$
G_{\theta, \lambda, \sigma}\left(x_{o}, y_{o}\right)=\left(I * g_{\theta, \lambda, \sigma}\right)\left(x_{o}, y_{o}\right)=\int(x, y) g_{\theta, \lambda, \sigma}\left(x_{o}-x, y_{o}-y\right) d x d y,(6)
$$

where* represents convolution.

The Gabor response obtained emphasized basically three types of characteristics in the image. The edge-oriented feature, texture-oriented feature and a combination of both. To emphasize different types of image characteristics the parameters $\sigma, \theta$, and $\lambda$ of the Gabor function are varied. The

variation of $\sigma$ changed the scale at which the world is being viewed, and the variation of $\lambda$, the selectivity to high/ low frequencies. An adequate combination of $\sigma, \theta$, and $\lambda$ were found to represent parts of objects for recognition task.

Large arrays of (high dimension) features were extracted from each subject using Gabor. However, since the dimension was too large and so unsuitable for classification, PCA was employed for feature dimensionality reduction, because it represents data in a low dimensional space while maintaining the Euclidean structures of the data in a high dimensional space. PCA reduced the dimension of features extracted via Gabor by selecting the most discriminative features that best represent the subject. $A$ total of ten (10) discriminative feature data was adopted for each subject. Support Vector Machine (SVM) learning algorithm was used to analyze data and recognize patterns to determine whether the gait of the subject being tested is available in the database or not. If the gait pattern of the tested subject matches the feature in the database the system accepts. If otherwise, the system rejects the tested subject. The algorithm was implemented in MATLAB 8.1.0.64 version and performance evaluation was done using the metrics of Accuracy, False Acceptance Rate, False Rejection Rate and Equal Error Rate.

Performance evaluation metrics used are:

1) Accuracy - the extent of reliability of the biometric system.

$$
\text { Accuracy }=(\text { Items classified correctly } \div \text { All classified items }) \times 100
$$


Ojo, John Adedapo, Afolabi, Simeon Olukayode; Gait Recognition System Using Gabor Wavelet and Active Gait Differential I mage. Advances in I mage and Video Processing, Volume 5 No 4, August (2017); pp: 1-10

2) False acceptance rate (FAR) - The proportion of impostors that are accepted by the biometric

system.

$$
\operatorname{FAR}(t)=\operatorname{FMR}(t) \times(1-\mathrm{FTA})
$$

3) False rejection rate (FRR) -The proportion of authentic users that are incorrectly denied,

$$
\operatorname{FRR}(t)=\mathrm{FTA}+\mathrm{FNMR}(t) \times(1-\mathrm{FTA})
$$

Equal error rate - this corresponds to the point at which the FAR and FRR crossed FAR $(t)=$ FMR $(t)$, which is the compromise between FAR and FRR.

\section{Results}

The performance evaluation results of the system as presented in Table 1, shows accuracy, FAR, FRR and EER of $99.19 \%, 1 \%, 0 \%$, and $2 \%$ respectively. The result of the performance of the developed method using Active Gait Differential Image (AGDI) and Gabor feature extraction technique over other feature image templates is presented in Table 2. The result in terms of accuracy of the developed AGDI feature extraction with Gabor filter method was compared with earlier methods as shown in Table 2.

Table 1 Performance Evaluation of the Proposed System

\begin{tabular}{|c|c|}
\hline Evaluation Metric & Result (\%) \\
\hline Accuracy & 99.19 \\
\hline Equal Error Rate & 1 \\
\hline False Rejection Rate & 2 \\
\hline False Acceptance Rate & 0 \\
\hline
\end{tabular}

Table 2: Comparison of new Feature Image Template and Existing Feature Images.

\begin{tabular}{|c|l|c|}
\hline SN & \multicolumn{1}{|c|}{ Feature Image Method } & Performance (\%) \\
\hline 1 & Gait Flow Image (GFI) [17] & 98 \\
\hline 2 & Enhanced Gait Energy Image [18] & 75 \\
\hline 3 & Frame Differential Energy Image (FDEI) [2] & 94 \\
\hline 4 & Structural Gait Differential Image (SGDI) [19] & 89.29 \\
\hline 5 & Gait Energy Image (GEI) [16] & 60.37 \\
\hline 6 & Gait Entropy Image (GEnI) [20] & 80.1 \\
\hline 7. & Developed Active Gait Differential Image (AGDI) & 99.19 \\
\hline
\end{tabular}

Gait flow image [17], Enhanced Gait Energy Image [18], Frame Differential Energy Image (FDEI) [2], Structural Gait Differential Image (SGDI) [19], Gait Energy Image (GEI) [19], Gait Entropy Image (GEnI) [20] gave $98 \%, 75 \%, 95 \%, 89.29 \%, 60.37 \%, 80.1 \%$ respectively as against $99.19 \%$ by the developed AGDI with Gabor filter method.

Table 3. Compares the accuracy of the proposed gait recognition system with existing silhouette-based gait recognition methods. Features were extracted on outermost contour of each silhouette image on a database of one hundred (100) people while employing Neural Network and Exepler Neural Network pattern classification techniques. The highest accuracy achieved in the combination of four (4) different techniques was $97.67 \%$ as against $99.19 \%$ accuracy of the developed system on a database of one hundred and twenty-four (124) people. Improved recognition rate was achieved due to the efficiency of AGDI and a more adequate feature extraction and classification methods employed in the experiment. 
Table 3: Comparison of results with existing Silhouette-Based Methods

\begin{tabular}{|l|c|c|c|}
\hline \multicolumn{1}{|c|}{ Method } & Classifier & Accuracy (\%) & Error rate (\%) \\
\hline PCA [21] & NN & 72.33 & 16 \\
\hline PCA+MDA [21] & NN & 96.67 & 8 \\
\hline PCA [21] & Exempler NN & 69 & 11 \\
\hline PCA+MDA [21] & Exempler NN & 97.67 & 5 \\
\hline PCA+Gabor (Developed system) & SVM & 99.19 & 1 \\
\hline
\end{tabular}

\section{Conclusion}

In this paper, we presented a gait recognition system that uses Gabor wavelet features extracted from Active Gait Differential Images and SVM as the classifier. The developed system gave accuracy, FAR, FRR and EER of $99.19 \%, 1 \%, 0 \%$, and $2 \%$ respectively, which shows some improvement over earlier methods. Future research in this area can be focused on gait images with various covariate conditions such as different view angles, clothing, carrying conditions (backpack, briefcase, and handbag), type of shoe worn, surface accessories, injury, and mood of the individuals.

\section{REFERENCES}

[1] Liang, W., Tieniu, T., Huazhong, Ning., and Weiming H., (2003) "Silhouette Analysis Based- Gait Recognition for Human Identification". IEEE Transactions on Pattern Analysis and Machine Intelligence 25, (12), pp. 1505-1518.

[2] Zhuowen, L.v., Xianglei, X., Kejun, W., and Donghai, G., (2015), “Class Energy Image Analysis for Video Sensor-Based Gait Recognition. Sensors, 2015, (15), pp. 932-964.

[3] Chiung, C.H., Eswaran, C., and June, Y.L., (2010), “An unobstructive Android person verification using Accelerometer based Gait". Proceedings of $10^{\text {th }}$ International conference on advances in mobile computing and multimedia. pp. 271-274.

[4] Johansson, G. (1976), "Spatio-temporal differentiation and integration in visual motion perception". Psychological Research, vol.38, pp 379-393.

[5] Huang, P.S., Harris, C. J., and Nixon, M.S., "Human gait Recognition in Canonical space using temporal template" IEEE Proceedings-Vision image signal process, Vol. 146, no. 2

[6] Michael, O., (2005) "Application of continuous wave radar for human gait recognition". The Proceedings of the SPIE, Volume 5809, pp. 538-548.

[7] Bobick, A.F. and Johnson, A.Y., "Gait recognition using static, activity-specific parameters," Proceedings of the 2001 IEEE Computer Society Conference on Computer Vision and Pattern Recognition. CVPR 2001, pp. I-423-I-430, vol.1.

[8] BenAbdelkader, C., Cutler. R and Davis. L., (2002) "Stride and cadence as a biometric in automatic person identification and verification". Fifth IEEE International Conference on Automatic Face and Gesture Recognition, 2002. pp. 372- 377.

[9] Murat, E.K., (2006) “Human Identification Using Gait". Computer Vision Lab." Turkish Journal of Electrical Engineering \& Computer Sciences, 14, (2), pp267-291. 
Ojo, John Adedapo, Afolabi, Simeon Olukayode; Gait Recognition System Using Gabor Wavelet and Active Gait Differential I mage. Advances in I mage and Video Processing, Volume 5 No 4, August (2017); pp: 1-10

[10] Goffredo, M., Carter, J.N., and Nixon, M.S., (2008) “Front-view Gait Recognition". 2 ${ }^{\text {nd }}$ IEEE International Conference on Biometrics: Theory, Applications and Systems, BTAS, Arlington, USA, pp. 1-6.

[11] Khalid B., Tao X., Shaogang G. (2008) "Feature selection on Gait Energy Image for Human Identification". IEEE International Conference on Acoustics, Speech and Signal Processing, ICASSP, Las Vegas, USA, April 2008, pp. 985-988.

[12] Chen C., Liang J., Zhao H., Hu H., Tian J. (2003): Frame difference energy image for gait recognition with incomplete silhouettes. Pattern Recognition. Lett. 30(11), 977-984

[13] Hayder, A., Jamal D., Chekima, A., and Ervin, G.M., (2011) “Gait Recognition using Gait Energy Image". International Journal of Sigal Processing, Image Processing and Pattern Recognition, 4 (3), pp. 141152.

[14] Sabesan, S., Daniel, C., Simon, D., Sridha, S., and Clinton, F., (2011), “Gait Energy Volumes and Frontal Gait Recognition using Depth Images". 2011 International Joint Conference on Biometrics (IJCB), Washington DC, USA, October 2011, pp.1-6.

[15] Yu, S., Tan, D. and Tan, T., 2006, August. A framework for evaluating the effect of view angle, clothing and carrying condition on gait recognition. In Pattern Recognition, 2006. ICPR 2006. 18th International Conference on 4, pp. 441-444, IEEE.

[16] Chen, J., and Liu, L., (2014), "Active Gait Differential Image Based Human Recognition”. The Scientific World Journal 2014, pp.1-8.

[17] Arora, P., Srivastava, S. and Singhal, S., 2016. Analysis of Gait Flow Image and Gait Gaussian Image Using Extension Neural Network for Gait Recognition. International Journal of Rough Sets and Data Analysis (IJRSDA), 3(2), pp.45-64.

[18] Iman. M.B., and Jan, N. (2015), “ Multiview Gait Recognition Using Enhanced Gait Energy Image and Radon Transform technique", Asian journal of applied science, 8 (2), pp 138-148.

[19] Xiaoxiang, L., and Youbing, C., (2013), "Gait Recognition based on Structural Gait Energy Image", Journal of computational information systems (2013) pp 121-126.

[20] Khalid B., Tao X., Shaogang G. (2009) "Gait Recognition using Gait Entropy Image", Department of computer science, 3rd International Conference on Crime Detection and Prevention (ICDP 2009), London, UK, Dec. 2009, pp. 1-6.

[21] Lili, L., Yilong Y., and Wei Q., (2011) “Gait recognition based on outermost contour". International Journal of Computational Intelligence Systems,4 (5), pp. 1090-1099.

[22] CASIA Gait Database. 2009, http://www.sinobiometrics.com 